\title{
On the Existence of an Universal Spin-down Equation for Pulsars
}

\author{
César Alvarez \& Alberto Carramiñana \\ Instiluto Nacional de Astrofísica Optica y Electrónica (INAOE), \\ Luis Enrique Erro No. 1 Tonantzintla Puebla, México. C.P. 72840
}

\begin{abstract}
The Taylor expansion of the generalized spin-down equation $\dot{\nu}=-f(\nu)$, derives into the multipolar equation $\dot{\nu}=-g \nu^{5}-r \nu^{3}-s \nu$, which is used here to study pulsar evolution. The coefficients $g, r$ and $s$ can be physically associated to gravitational radiation, magnetic dipolar radiation and particle acelerat ion. The multipolar expansion with constant coefficients cannot describe the $P \dot{P}$ diagram in a consistent manner and therefore it is unlikely a generalized equation of the form $\dot{\nu}=-f(\nu)$ can be used to describe pulsar evolution. A more general form, $\dot{\nu}=-f(\nu ; t)$ can be investigated giving to the coefficients $g, r$ and $s$ time dependence. We consider some explicit functional forms for $g(t), r(t)$ and $s(t)$ which are consistent with observational and theoretical constraints.
\end{abstract}

\section{Introduction}

How pulsars evolve from the young population into the average old population remains an open question. It is believed that pulsars are born with short periods and high period derivatives in Supernova explosions. This standard model stablishes that pulsar spin-down like a magnetic dipole, implying a braking index $n=3$. The four measured braking indices are below this predicted value (Kaspi et al. 1994; Boyd et al. 1995). We consider a general spin-down equation, $\dot{\nu}=-f(\nu, t)$, which can be expanded in multipole terms, which takes into account the dipole magnetic and quadrupole energy radiation losses, together with particle acceleration.

\section{Mathematical form for the Spin-Down equation}

We start from a general form for the rate change of frequency pulsar's rotation, $\dot{\nu}=-f(\nu, t)$. Energy loss implies that $f>0$ for all $(\nu, t)$. A sign can be ascribed to the frequency and its derivative, $\nu$ and $\dot{\nu}$, in order to account for the orientation $(\nu)$ and spin-down versus spin-up $(\dot{\nu})$. From th is we can see that the general spin-down equation must be antisymmetric, $f(-\nu)=-f(\nu)$, implying a Taylor expansion in odd terms only, which we restrict to order below $\nu^{7}: f(\nu)=s(t) \nu+r(t) \nu^{3}+g(t) \nu^{5}$. The second term $\left(r(t) \nu^{3}\right)$ can be associated with magnetic dipole losses and $g(t) \nu^{5}$ with quadrupolar radiation terms, like gravitational radiation. We propose the association of the linear term $(s(t) \nu)$ with particle acceleration. Note that onl y the existence of this term can produce 


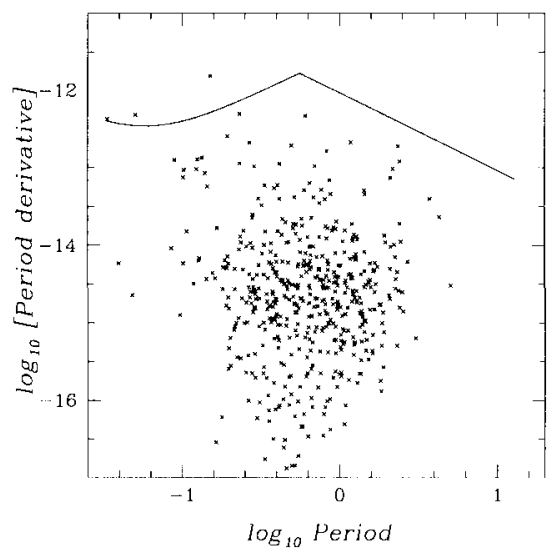

Figure 1. A typical evolutionary $P \dot{P}$ trajectory for the Crab pulsar.

braking indices below 3 , as measured. Time dependences of the coefficients $g, r, s$ are physically related to time behaviour of the magnetic field $B$, figure ellipticity $e$ and an efficiency parameter $\eta$ which we introduce in the monopolar term.

We previously studied the $\dot{\nu}=-f(\nu)$ equation with constant coefficients $g, r$ and $s$, obtaining trajectories incompatible with the $P \dot{P}$ diagram (Alvarez \& Carramiñana 1998). We need to introduce time dependences compatible with physical processes like the magnetic field (Bhattacharya 1992), gravitational emission (New et al. 1995) and the $L_{\gamma}$ versus age relation for EGRET pulsars (Thompson 1994). In particular, dimensional considerations lead us to the expression, $s(t)=\eta(t)\left(2 B^{2}(t) R^{4} / 3 I c\right)$, for the monopolar coefficient. $\eta(t)$ is a dimensionless particle acceleration efficiency, with step function modulation -to account for the suppression of particle acceleration after a threshold pulsar age.

\section{Results and conclusions}

Figure 1 shows a typical evolutionary trajectory for the Crab from its present parameters, assuming $r$ and $g$ to constant. The monopolar term is suppressed when $n \approx 1$, at a point clearly noticeable in the Figure. The trajectory does not lead to $\mathrm{t}$ he main pulsar population and is still incompatible with the overall distribution in the $P \dot{P}$ diagram.

\section{References}

Alvarez, C., Carramiñana, A. 1998, RevMexAASC, 7, 207

Bhattacharya D., Wijers R.A.M.J., Hartman J.W., Verbunt F. 1992, A\&A254,198

Boyd, P. T. et al. 1995, ApJ, 448, 365

Kaspi V.M., et al. 1994, ApJ422, L83

Lyne, A.G., Pritchard, R.S., \& Smith, F.G. 1993, MNRAS, 265, 1003

Lyne, A. G., Pritchard, R. S., Smith, F. G., \& Camilo, F. 1996 Nature, 381, 497

New K.C.B., Chanmugam G., Johnson W.W., Tohline J.E. 1995, ApJ450, 757

Thompson D.J., et. al. 1994, ApJ, 436, 229 